\begin{tabular}{|c|l|}
\hline Title & Kinetic Boundary Condition at a V apor-Liquid Interface \\
\hline Author(s) & Ishiyama, Tatsuya; Y ano, Takeru; Fujikawa, Shigeo \\
\hline Citation & $\begin{array}{l}\text { Physical Review Letters, 95(8), 084504 } \\
\text { https://doi.org/40.1103/PhysRevLett.95.084504 }\end{array}$ \\
\hline Issue Date & 2005-08 \\
\hline Doc URL & http://hdl.handle.net/2115/16946 \\
\hline Rights & Copyright $\odot$ 2005 A merican Physical Society \\
\hline Type & article \\
\hline File Information & PRL95-084504.pdf \\
\hline
\end{tabular}

Instructions for use 


\title{
Kinetic Boundary Condition at a Vapor-Liquid Interface
}

\author{
Tatsuya Ishiyama, Takeru Yano, and Shigeo Fujikawa \\ Division of Mechanical Science, Graduate School of Engineering, Hokkaido University, Sapporo 060-8628, Japan
}

(Received 4 December 2004; published 19 August 2005)

\begin{abstract}
By molecular dynamics simulations, the boundary condition for the Boltzmann equation at a vaporliquid interface is found to be the product of three one-dimensional Maxwellian distributions for the three velocity components of vapor molecules and a factor including a well-defined condensation coefficient. The Maxwellian distribution for the velocity component normal to the interface is characterized by the liquid temperature, as in a conventional model boundary condition, while those for the tangential components are prescribed by a different temperature, which is a linear function of energy flux across the interface. The condensation coefficient is found to be constant and equal to the evaporation coefficient determined by the liquid temperature only.
\end{abstract}

PACS numbers: 51.10.+y, 47.45. $-\mathrm{n}$

In fluid dynamics and kinetic theory of gases, the boundary condition is of great importance because it has relevance to the drag and lift exerted on the body and heat and mass transfers across the boundary. While the governing equations (the Navier-Stokes equations in fluid dynamics and the Boltzmann equation in kinetic theory) can be derived from macroscopic or microscopic conservation laws, the derivation of boundary condition sometimes requires further detailed information of molecular phenomena at the boundary. Molecular dynamics (MD) can serve this purpose. In fact, recent studies on the slip conditions at fluid-solid boundaries have made significant progress using MD simulations [1-4].

In this Letter, using MD simulations, we present a new kinetic boundary condition (KBC) for the Boltzmann equation at the interface between argon vapor and its condensed phase. Since the KBC at the interface is associated with the mass flux across the interface (evaporation or condensation), it has crucial relevance to the behavior of the gas as compared with the $\mathrm{KBC}$ at a gas-solid boundary, across which there is no mass flux. The physically correct form of the KBC at the interface has been unknown and the only model available and believed reliable is (at an interface at rest) $[5,6]$

$$
\begin{gathered}
f^{\text {out }}=\left[\alpha \rho_{v}+(1-\alpha) \sigma_{w}\right] \hat{f}^{*}\left(T_{\ell}\right) \quad\left(\xi_{z}>0\right), \\
\sigma_{w}=-\sqrt{\frac{2 \pi}{R T_{\ell}}} \int_{\xi_{z}<0} \xi_{z} f^{\text {coll }} d \xi,
\end{gathered}
$$

where $f^{\text {out }}$ is the velocity distribution function of molecules leaving the interface, $\rho_{v} \hat{f}^{*}\left(T_{\ell}\right)=\rho_{v} \exp \left[-\left(\xi_{x}^{2}+\right.\right.$ $\left.\left.\xi_{y}^{2}+\xi_{z}^{2}\right) /\left(2 R T_{\ell}\right)\right] /\left[\left(2 \pi R T_{\ell}\right)^{3 / 2}\right]$ is the equilibrium distribution (Maxwellian) with the saturated vapor density $\rho_{v}$ at the temperature of the condensed phase $T_{\ell}, f^{\text {coll }}$ is the distribution of molecules incident on the interface, $\xi_{x}$ and $\xi_{y}$ are the molecular velocity components tangential to the interface, $\xi_{z}$ is the normal component, $R$ is the gas constant per unit mass, and $\alpha$ is a parameter between zero and unity. The simpleness of conventional model (1) with
(2) is suitable for analytical studies and a number of problems have been solved with this model (see Refs. [5,6] and references therein).

However, Eq. (1) assumes, without verification, that $f^{\text {out }}$ is in proportion to $\hat{f}^{*}\left(T_{\ell}\right)$. On this assumption, the lack of necessary information of phenomena at the interface is augmented by the introduction of parameter $\alpha$; the mass flux across the interface is then given as $\alpha\left(\rho_{v}-\sigma_{w}\right) \times$ $\sqrt{R T_{\ell} /(2 \pi)}$. Historically, $\alpha$ has been called the condensation coefficient and its value, e.g., for water, has excited much controversy (see, for example, Ref. [7]). Unless the validity of Eq. (1) is proven, however, such controversy may be unfruitful. Although some authors recently addressed the $\mathrm{KBC}$ at the interface with MD methods [811 ], they could not reach the physically correct KBC owing to their ambiguous definitions of the evaporation and reflection of molecules, as criticized in Refs. [12,13].

In the kinetic theory, the velocity distribution $f^{\text {out }}$ should be a function of the temperature at the interface $T_{\ell}$ and the velocity distribution of molecules incident on it $f^{\text {coll }}$, in addition to the molecular velocity $\xi$, i.e., $f^{\text {out }}=$ $f^{\text {out }}\left(\boldsymbol{\xi}, T_{\ell}, f^{\text {coll }}\right)$. We therefore investigate $f^{\text {out }}\left(\xi, T_{\ell}, f^{\text {coll }}\right)$ for various $f^{\text {coll }}$ and a specified $T_{\ell}=85 \mathrm{~K}$ (near the triplepoint of argon) in nonequilibrium steady MD simulations for argon vapor-liquid two-phase system, thereby constructing the physically correct form of $\mathrm{KBC}$ at the interface for argon. This may be regarded as an extension of our previous studies of vacuum evaporation simulation $[12,13]$, where we have examined $f^{\text {out }}\left(\xi, T_{\ell}, 0\right)$, since $f^{\text {coll }}=0$ when the liquid phase is in contact with vacuum.

The phenomenon considered is the one-dimensional steady evaporation or condensation flow in the macroscopic sense. The MD computations are executed in the following way: a rectangular simulation cell with dimensions $L_{x} \times L_{y} \times L_{z}$ is used, the lateral dimensions of which are $L_{x}=L_{y}=50 \AA$, and the initial $L_{z}$ is $88.3 \AA$, which is changed as explained later. The cell is initially filled with $N=2000$ argon molecules in a vapor-liquid equilibrium state at $85 \mathrm{~K}$. The liquid phase forms a planar 
liquid layer, parallel to the $x y$ plane, with thickness $38.2 \AA$ at the center of the cell. The 12-6 Lennard-Jones potential $\phi\left(r_{i j}\right)=4 \epsilon\left[\left(\sigma / r_{i j}\right)^{12}-\left(\sigma / r_{i j}\right)^{6}\right]$ is applied for the intermolecular potential of argon molecules, where $\sigma=$ $3.405 \AA, \epsilon / k=119.8 \mathrm{~K}$ ( $k$ is the Boltzmann constant), and $r_{i j}$ is the intermolecular separation. Newton's equations of motion for $N$ molecules are integrated numerically using the leapfrog algorithm with a time step $1 \mathrm{fs}$. The periodic boundary condition is imposed at the cell faces in the $\pm x$ and $\pm y$ directions, while the boundary condition at the cell faces in the $\pm z$ directions and the cell length $L_{z}$ are treated specially (see below).

For the boundary condition at the cell faces in the $\pm z$ direction (hereafter referred to as top and bottom faces, respectively), we utilize an algorithm widely used in the direct simulation Monte_Carlo method [14]. That is, we give the positions and velocities of molecules incoming to the interface across the top or bottom face probabilistically with the uniform random number $R_{n}\left(0 \leq R_{n} \leq 1\right)$, and eliminate the molecules outgoing across these boundaries. The position of the $i$ th molecule coming into the cell is determined as $\left(x^{i}, y^{i}\right)=\left(L_{x} R_{1}, L_{y} R_{2}\right)$ in the top or bottom face. To avoid unphysical overlap of intermolecular potentials, the position $\left(x^{i}, y^{i}\right)$ is rejected if $r_{i j}<\sigma$ for some $j$ th molecule, and the random numbers are regenerated until $r_{i j}>\sigma$ is satisfied for any other $j$ th molecules. The velocities of molecules incoming to the interface are given according to the direct method [14], on the basis of a specified distribution function $f^{\text {coll }}$, for which we assume the Maxwell type distribution function,

$$
f^{\text {coll }}=\frac{\beta \rho_{v}}{\left(2 \pi R \gamma T_{\ell}\right)^{3 / 2}} \exp \left(-\frac{\xi_{x}^{2}+\xi_{y}^{2}+\xi_{z}^{2}}{2 R \gamma T_{\ell}}\right),
$$

for $\xi_{z}<0$ on the top face and for $\xi_{z}>0$ on the bottom one. Here, $\beta$ and $\gamma$ are parameters that represent the deviation from the equilibrium state. The equilibrium state corresponds to $\beta=\gamma=1$, and the vacuum evaporation state [12] is realized when $\beta=0$. We present the results of numerical simulations for 16 different sets of $\beta=1,2,3,4$ and $\gamma=1,2,3,4$. Except for the case of $\beta=\gamma=1$, the net condensation occurs since the temperature and pressure in the vapor phase become high compared with those in the equilibrium state. Note that the compression factor $p /(\rho R T)$ is confirmed to be nearly unity in all cases, and hence the vapor can be regarded as an ideal gas. In a high temperature case, e.g., $T_{\ell}=100 \mathrm{~K}$ for argon, the ideal-gas approximation becomes inaccurate, and the vapor may not be considered as in the Grad-Boltzmann limit $[5,6]$.

From now, we focus on the dynamics of molecules in the upper half region in the cell because the vapor-liquid system is symmetric with respect to the center of the liquid layer in the macroscopic sense. When the net condensation occurs, the thickness of the liquid layer increases with time and the interface moves towards the vapor phase. We therefore introduce the moving coordinate system, as in the previous study [12], $z^{*}=\left[z-\left(Z_{m}-v_{s} t\right)\right] / \delta, v_{s}=J_{s} / \rho_{\ell}$, where $Z_{m}$ and $\delta$ are, respectively, the center position and the $10-90$ thickness of the transition layer $(6.3 \AA)$, which have already been obtained in the equilibrium simulation [12], $v_{s}$ is the speed of the moving coordinate, $t$ is the time from the beginning of the simulation, $\rho_{\ell}$ is the density in the liquid phase, and $J_{s}$ is the nonaveraged net mass flux across the top face. To realize a steady condensation state, the distance between the top face and $Z_{m}$ should be unchanged during the simulation. The cell length $L_{z}$ is therefore changed so as to keep the distance. The liquid layer is thermostatted at $T_{\ell}$ with the velocity scaling method [15]. After a steady state is established, samples are accumulated throughout the simulation time of several tens of nanoseconds, and statistical averages are calculated on the $z^{*}$ coordinate from tens of millions of samples.

Figure 1 shows the profiles of averaged density, velocity, and temperature on the $z^{*}$ coordinate in the steady states. In the case of $\beta=\gamma=1$, the vapor-liquid equilibrium is realized, i.e., $\rho=\rho_{v}, v=0$, and $T=T_{\ell}$. For $\beta=\gamma=$ 2 and 4, the vapors have higher densities than $\rho_{v}$ and negative velocities, which means net condensation states. What is important in Fig. 1 is that the profiles of averaged density, velocity, and temperature are almost flat in the range $2<z^{*}<4$ of width $2 \delta$, in spite the fact that the vapor is not in a local equilibrium state. This suggests that the molecular collisions rarely happen there. In fact, the Knudsen numbers estimated by $\mathrm{Kn}=\ell /(2 \delta)=$ $1 /\left[\sqrt{2} \pi \sigma^{2}(\rho / m) 2 \delta\right]$ are large $(\ell$ is the mean free path of the vapor molecules, $m$ is the mass of argon molecule):
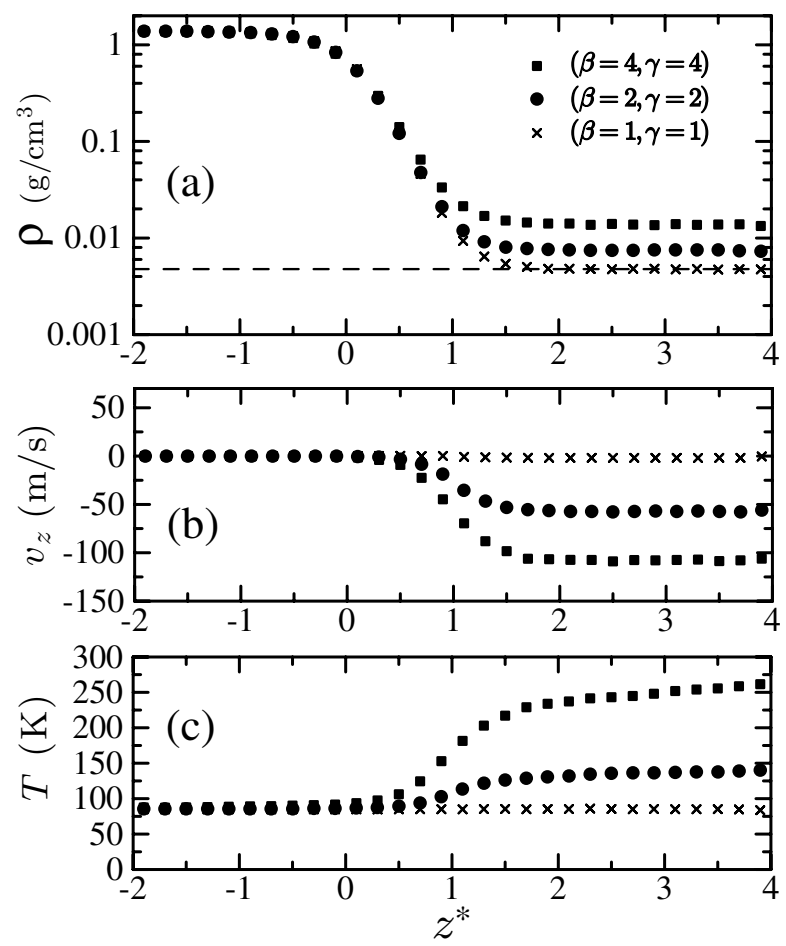

FIG. 1. Density, velocity, and temperature distributions for some cases of $(\beta, \gamma)$. The dashed line in (a) denotes the saturated vapor density $\rho_{v}$ for $T_{\ell}=85 \mathrm{~K}: \rho_{v}=4.59 \times 10^{-3} \mathrm{~g} / \mathrm{cm}^{3}$. 
$\mathrm{Kn}=20.9,13.5$, and 7.3 for $\beta=\gamma=1,2$, and 4, respectively. Since the thickness of transition layer $\delta$ is regarded as zero in the kinetic theory and the change in the vapor condition in the range $2<z^{*}<4$ is negligible, the kinetic interface may be located at an arbitrary position in this range. We therefore evaluate $f^{\text {out }}$ by sampling the molecular velocity in $2<z^{*}<4$.

To determine $f^{\text {out }}$ as a function of $\boldsymbol{\xi}$, it is useful to examine a normalized function $\hat{f}^{\text {out }}=f^{\text {out }} /\left(\int_{\xi_{z}>0} f^{\text {out }} d \xi\right)$. After the functional form of $\hat{f}^{\text {out }}$ is obtained, we elucidate $\int_{\xi_{z}>0} f^{\text {out }} d \boldsymbol{\xi}$, and thus the construction of $f^{\text {out }}$ is completed. In Fig. 2, we plot the marginal distributions, $\hat{f}_{z}=$ $\int_{\xi_{y}} \int_{\xi_{x}} \hat{f}^{\text {out }} d \xi_{x} d \xi_{y}$ and $\hat{f}_{x}=\int_{\xi_{z}>0} \int_{\xi_{y}} \hat{f}^{\text {out }} d \xi_{y} d \xi_{z}$, where $\hat{f}_{x}=\hat{f}_{y}$ due to the symmetry. Note that the marginal distributions are obtained not by integrating $\hat{f}^{\text {out }}$, which is unknown at this stage, but by sampling the molecular velocity in MD simulations. As shown in Figs. 2(a)-2(d), the distribution of velocity component normal to the interface is uniquely determined as the half-Maxwellian distribution with temperature $T_{\ell}, \sqrt{2 R T_{\ell}} \hat{f}_{z}=(2 / \sqrt{\pi}) \exp \left(-\zeta_{z}^{2}\right)$, for various $f^{\text {coll }}$, where $\zeta_{i}=\xi_{i} / \sqrt{2 R T_{\ell}}(i=x, y, z)$. On the other hand, the distributions of tangential component shown in Figs. 2(e) $-2(\mathrm{~h})$ vary with $f^{\text {coll }}$, retaining the functional form of one-dimensional normalized Maxwellian distribution with temperature $T_{t}, \sqrt{2 R T_{\ell}} \hat{f}_{x}=$ $\sqrt{T_{\ell} /\left(T_{t} \pi\right)} \exp \left(-\zeta_{x}^{2} T_{\ell} / T_{t}\right)$. In the figures, $T_{t}=(1 / R) \times$ $\int_{\xi_{z}>0} \xi_{x}^{2} \hat{f}^{\text {out }} d \xi$ is calculated in the MD simulation.
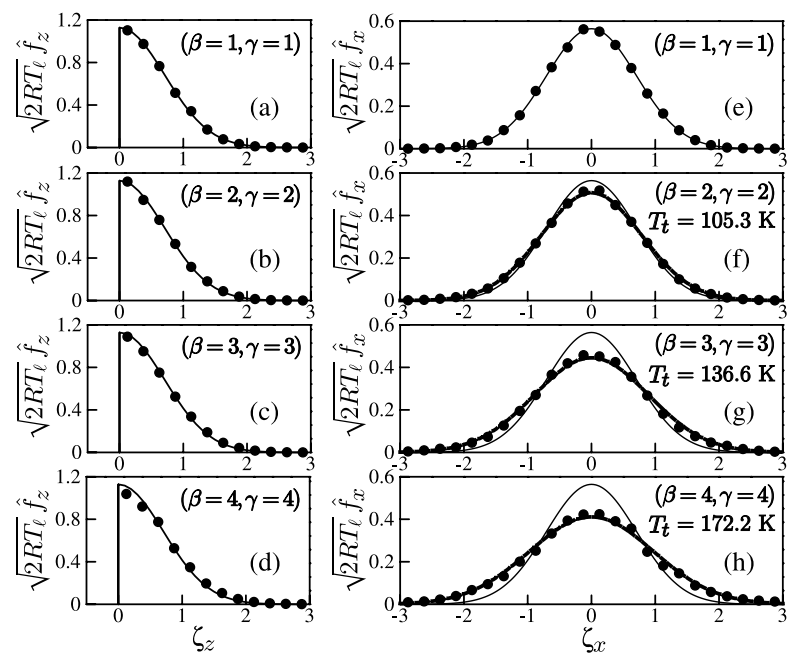

FIG. 2. Marginal distributions of vapor molecules outgoing from the interface, where $\zeta_{i}=\xi_{i} / \sqrt{2 R T_{\ell}}(i=x, z)$. The thin solid curves in (a)-(d) denote a one-dimensional normalized half-Maxwellian distribution with $T_{\ell}(=85 \mathrm{~K}):(2 / \sqrt{\pi}) \times$ $\exp \left(-\zeta_{z}^{2}\right)\left(\zeta_{z}>0\right)$, and those in (e)-(h) a one-dimensional normalized Maxwellian distribution with $T_{\ell}:(1 / \sqrt{\pi}) \exp \left(-\zeta_{x}^{2}\right)$. The bold solid curves in (e)-(h) are one-dimensional normalized Maxwellian distributions with different temperatures $T_{t}$ : $\sqrt{T_{\ell} /\left(T_{t} \pi\right)} \exp \left(-\zeta_{x}^{2} T_{\ell} / T_{t}\right)$.
Figures 2(e)-2(h) also show that $T_{t}$ becomes higher and higher as $\beta$ and $\gamma$ increase.

Figure 3 shows that $T_{t}$ is a linear function of $\left\langle E_{\text {coll }}\right\rangle-$ $\left\langle E_{\text {coll }}\right\rangle_{\mathrm{e}}$, where $\left\langle E_{\text {coll }}\right\rangle=\int_{\xi_{z}<0}\left[\left(\xi_{x}^{2}+\xi_{y}^{2}+\xi_{z}^{2}\right) / 2\right] \xi_{z} f^{\text {coll }} d \xi$ is the energy flux of vapor molecules incoming across the interface and $\left\langle E_{\text {coll }}\right\rangle_{\mathrm{e}}=\int_{\xi_{z}<0}\left[\left(\xi_{x}^{2}+\xi_{y}^{2}+\xi_{z}^{2}\right) / 2\right] \times$ $\xi_{z} \rho_{v} \hat{f}^{*}\left(T_{\ell}\right) d \xi=\rho_{v} \sqrt{2\left(R T_{\ell}\right)^{3} / \pi}$ is its counterpart in the equilibrium state. As can be seen, $T_{t}$ is equal to $T_{\ell}$ in the equilibrium state and increases with increase in $\left\langle E_{\text {coll }}\right\rangle$. The result of vacuum simulation [12] is indicated by an open circle near the equilibrium, and hence we predict that all the evaporation states may be included in the present result consistently. We have also examined the relations between $T_{t}$ and the mass and momentum fluxes of incoming vapor molecules, and evidenced that there are no definite relations between them.

The joint distribution $\hat{f}^{\text {out }}$ is the product of the marginal distributions when $\xi_{x}, \xi_{y}$, and $\xi_{z}$ are the independent random variables. As a check of a necessary condition for the statistical independence, we have confirmed that the correlation matrix is nearly diagonal, and therefore $\hat{f}^{\text {out }}$ may be expressed as $\hat{f}^{\text {out }}=\hat{f}_{x} \hat{f}_{y} \hat{f}_{z}$. Since the functional form of each marginal distribution is found to be the Maxwellian distribution with $T_{t}$ or the half-Maxwellian distribution with $T_{\ell}$ as shown in Fig. 2, we have

$$
f^{\text {out }}=\frac{\rho_{\text {out }}}{(2 \pi R)^{3 / 2} T_{\ell} \sqrt{T_{\ell}}} \exp \left(-\frac{\xi_{x}^{2}+\xi_{y}^{2}}{2 R T_{t}}-\frac{\xi_{z}^{2}}{2 R T_{\ell}}\right),
$$

for $\xi_{z}>0$, where $\rho_{\text {out }}=2 \int_{\xi_{z}>0} f^{\text {out }} d \xi$ (the factor 2 comes from $\hat{f}_{z}$ ).

The KBC should give the relation between $f^{\text {out }}$ and $f^{\text {coll }}$. We have already demonstrated in Fig. 3 that $T_{t}$ in Eq. (4) is the linear function of the incoming energy flux. Accordingly, the final stage of the construction of the $\mathrm{KBC}$ at the interface is to connect the factor $\rho_{\text {out }}$ in Eq. (4) to $f^{\text {coll }}$. As shown in the following, $\rho_{\text {out }}$ is affected by $f^{\text {coll }}$ only through the incoming mass flux, $\left\langle J_{\text {coll }}\right\rangle=$ $-\int_{\xi_{z}<0} \xi_{z} f^{\text {coll }} d \xi$. To prove this, we split $f^{\text {out }}$ into two

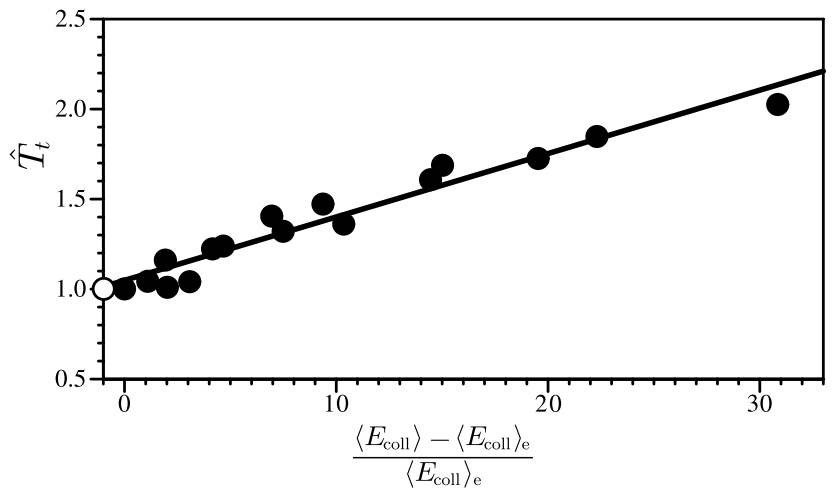

FIG. 3. The reduced temperature $\hat{T}_{t}=T_{t} / T_{\ell}$ as a function of the energy flux of incoming molecules across the interface. The open circle denotes the result of vacuum simulation [12]. 


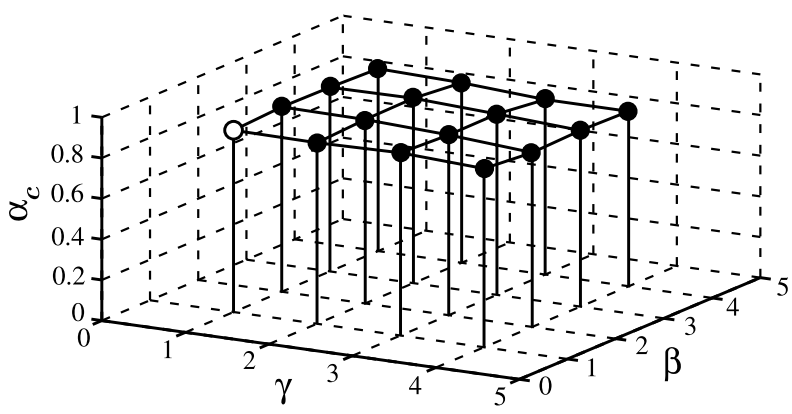

FIG. 4. The condensation coefficient $\alpha_{c}$ in nonequilibrium states. The open circle denotes the equilibrium state, where $\alpha_{c}$ is equal to the evaporation coefficient $\alpha_{e}$ from their definitions.

parts, i.e., $f^{\text {out }}\left(T_{\ell}, f^{\text {coll }}\right)=f^{\text {out }}\left(T_{\ell}, 0\right)+\left[f^{\text {out }}\left(T_{\ell}, f^{\text {coll }}\right)-\right.$ $\left.f^{\text {out }}\left(T_{\ell}, 0\right)\right]$. The first term is independent of $f^{\text {coll }}$ and is called the spontaneous evaporation [12]. All the effects of $f^{\text {coll }}$ are confined into the second term, and hence it may be called the reflection. This is the only splitting that discriminates the evaporation from the reflection without ambiguity [12]. The previous authors [8-11] tried to classify individual outgoing molecules as either evaporated or reflected one with artificial criterions. However, the complete classification of individual molecules may be impossible, because the reflected molecules in many cases condense into the inside of the interface before being reemitted.

According to the above splitting, the outgoing mass flux $\left\langle J_{\text {out }}\right\rangle=\int_{\xi_{z}>0} \xi_{z} f^{\text {out }} d \boldsymbol{\xi}$ can also be split into $\left\langle J_{\text {out }}\right\rangle=$ $\left\langle J_{\text {evap }}\right\rangle+\left\langle J_{\text {ref }}\right\rangle$, where $\left\langle J_{\text {evap }}\right\rangle=\int_{\xi_{z}>0} \xi_{z} f^{\text {out }}\left(T_{\ell}, 0\right) d \xi$ and $\left\langle J_{\text {ref }}\right\rangle=\int_{\xi_{z}>0} \xi_{z}\left[f^{\text {out }}\left(T_{\ell}, f^{\text {coll }}\right)-f^{\text {out }}\left(T_{\ell}, 0\right)\right] d \xi$. From the balance of the mass fluxes across the interface, the difference between the incoming mass flux $\left\langle J_{\text {coll }}\right\rangle$ and the reflection mass flux $\left\langle J_{\text {ref }}\right\rangle$ should condense onto the interface. We can therefore define the condensation mass flux as $\left\langle J_{\text {cnds }}\right\rangle=\left\langle J_{\text {coll }}\right\rangle-\left\langle J_{\text {ref }}\right\rangle$. These relations enable us to rewrite $\left\langle J_{\text {out }}\right\rangle$ into $\left\langle J_{\text {out }}\right\rangle=\left\langle J_{\text {evap }}\right\rangle+\left\langle J_{\text {coll }}\right\rangle\left(1-\left\langle J_{\text {cnds }}\right\rangle /\right.$ $\left.\left\langle J_{\text {coll }}\right\rangle\right)$. Furthermore, since Eq. (4) yields $\left\langle J_{\text {out }}\right\rangle=$ $\rho_{\text {out }} \sqrt{R T_{\ell} /(2 \pi)}$, we can eliminate $\left\langle J_{\text {out }}\right\rangle$ to give

$$
\rho_{\text {out }}=\frac{\left\langle J_{\text {evap }}\right\rangle}{\sqrt{R T_{\ell} /(2 \pi)}}+\left(1-\frac{\left\langle J_{\text {cnds }}\right\rangle}{\left\langle J_{\text {coll }}\right\rangle}\right) \frac{\left\langle J_{\text {coll }}\right\rangle}{\sqrt{R T_{\ell} /(2 \pi)}} .
$$

This naturally leads to the introduction of the evaporation coefficient $\alpha_{e}$ and the condensation coefficient $\alpha_{c}$ defined as [12],

$$
\alpha_{e}=\frac{\left\langle J_{\text {evap }}\right\rangle}{\rho_{v} \sqrt{R T_{\ell} /(2 \pi)}}, \quad \alpha_{c}=\frac{\left\langle J_{\text {cnds }}\right\rangle}{\left\langle J_{\text {coll }}\right\rangle},
$$

and by using Eq. (2), we obtain $\rho_{\text {out }}=\alpha_{e} \rho_{v}+(1-$ $\left.\alpha_{c}\right) \sigma_{w}$. In Ref. [12], we have studied $\left\langle J_{\text {evap }}\right\rangle$ and $\alpha_{e}$ by the vacuum evaporation simulation for argon, and demonstrated that $\alpha_{e}$ is a function of the temperature in the condensed phase alone.

The condensation coefficient $\alpha_{c}$ evaluated with the use of $\left\langle J_{\text {cnds }}\right\rangle=\left\langle J_{\text {coll }}\right\rangle-\left(\left\langle J_{\text {out }}\right\rangle-\left\langle J_{\text {evap }}\right\rangle\right)$ is plotted in Fig. 4, which clearly shows that $\alpha_{c}$ is almost equal to $\alpha_{e}=0.868$ at $T_{\ell}=85 \mathrm{~K}$ [12]. We emphasize that if $\alpha_{c}$ is a constant, it must be equal to $\alpha_{e}$. Consequently, from Eqs. (4)-(6), the physically correct $\mathrm{KBC}$ at the interface is determined as

$$
f^{\text {out }}=\frac{\left[\alpha \rho_{v}+(1-\alpha) \sigma_{w}\right]}{(2 \pi R)^{3 / 2} T_{t} \sqrt{T_{\ell}}} \exp \left(-\frac{\xi_{x}^{2}+\xi_{y}^{2}}{2 R T_{t}}-\frac{\xi_{z}^{2}}{2 R T_{\ell}}\right)
$$

for $\xi_{z}>0$, where we have used the result in Fig. 4, i.e., $\alpha=\alpha_{e}=\alpha_{c}$. It is obvious that conventional model (1) with (2) is recovered in the limit of the equilibrium state, i.e., $T_{t}=T_{\ell}$.

In summary, using steady nonequilibrium MD simulations of vapor-liquid two-phase system of argon, we construct the physically correct $\mathrm{KBC}$ at the vapor-liquid interface in the form of Eq. (7). The new KBC is similar to the conventional model in the sense that $f^{\text {out }}$ is the product of one-dimensional Maxwellian distributions. However, it does not contain any arbitrary parameters: $\alpha$ should be the evaporation coefficient defined in Eq. (6), and $T_{t}$ is the linear function of $\left\langle E_{\text {coll }}\right\rangle$ as verified in Fig. 3. In particular, Fig. 4 will bring the long lasting controversy over $\alpha$ to an end.

[1] P. A. Thompson and S. M. Troian, Nature (London) 389 , 360 (1997).

[2] M. Cieplak, J. Koplik, and J. R. Banavar, Phys. Rev. Lett. 86, 803 (2001).

[3] C. Denniston and M. O. Robbins, Phys. Rev. Lett. 87, 178302 (2001).

[4] T. Qian, X. P. Wang, and P. Sheng, Phys. Rev. Lett. 93, 094501 (2004).

[5] Y. Sone, Kinetic Theory and Fluid Dynamics (Birkhäuser, Boston, 2002).

[6] C. Cercignani, Rarefied Gas Dynamics (Cambridge University Press, Cambridge, England, 2000).

[7] R. Marek and J. Straub, Int. J. Heat Mass Transf. 44, 39 (2001).

[8] G. Nagayama and T. Tsuruta, J. Chem. Phys. 118, 1392 (2003).

[9] A. Frezzotti, P. Grosfils, and S. Toxvaerd, Phys. Fluids 15, 2837 (2003)

[10] A. Morita, M. Sugiyama, H. Kameda, S. Koda, and D. R. Hanson, J. Phys. Chem. B 108, 9111 (2004).

[11] R. Meland, A. Frezzotti, T. Ytrehus, and B. Hafskjold, Phys. Fluids 16, 223 (2004).

[12] T. Ishiyama, T. Yano, and S. Fujikawa, Phys. Fluids 16, 2899 (2004).

[13] T. Ishiyama, T. Yano, and S. Fujikawa, Phys. Fluids 16, 4713 (2004).

[14] G. A. Bird, Molecular Gas Dynamics and the Direct Simulation of Gas Flows (Oxford University Press, Oxford, 1994).

[15] M.P. Allen and D. J. Tildesley, Computer Simulation of Liquids (Clarendon Press, Oxford, 1987). 